\title{
Universal analytic preorders arising from surjective functions
}

\author{
by \\ Riccardo Camerlo (Torino)
}

\begin{abstract}
Examples are presented of $\boldsymbol{\Sigma}_{1}^{1}$-universal preorders arising by requiring the existence of particular surjective functions. These are: the relation of epimorphism between countable graphs; the relation of being a continuous image (or a continuous image of some specific kind) for continua; the relation of being continuous open image for dendrites.
\end{abstract}

Introduction. Let $R, R^{\prime}$ be $n$-ary relations on standard Borel spaces $X, X^{\prime}$, respectively. Then $R$ Borel reduces to $R^{\prime}$, in symbols $R \leq_{B} R^{\prime}$, if and only if there is a Borel function $\varphi: X \rightarrow X^{\prime}$ such that

$$
\forall x_{1}, \ldots, x_{n} \in X\left(R\left(x_{1}, \ldots, x_{n}\right) \Leftrightarrow R^{\prime}\left(\varphi\left(x_{1}\right), \ldots, \varphi\left(x_{n}\right)\right)\right) .
$$

For each $n$, the relation $\leq_{B}$ is a preorder among $n$-ary relations on standard Borel spaces and it has been extensively studied for some classes of finitary relations (for example, for $n=1 ; n=2$ and $R, R^{\prime}$ equivalence relations; $n=2$ and $R, R^{\prime}$ preorders). The purpose of this paper is to present examples of universal analytic preorders of a particular kind. An analytic preorder on the standard Borel space $X$ is a preorder $R$ on $X$ that is analytic $\left(\boldsymbol{\Sigma}_{1}^{1}\right)$ as a subset of $X^{2}$. It is universal if and only if, for every analytic preorder $S$ on a standard Borel space, the relation $S \leq_{B} R$ holds. The existence of universal analytic preorders is proved in [LR05]. If $R, S$ are analytic preorders, $R$ is universal and $R \leq_{B} S$, then $S$ is universal as well.

Since analytic sets are closed under projections, a way to generate an analytic binary relation $R$ is to define $x R x^{\prime}$ if and only if there exists some $f$, ranging in a standard Borel space, such that $B\left(f, x, x^{\prime}\right)$ where $B$ is also analytic (or Borel). If for each $x$ there is an element $\operatorname{id}_{x}$ granting $B\left(\mathrm{id}_{x}, x, x\right)$ and given $f_{x x^{\prime}}, f_{x^{\prime} x^{\prime \prime}}$ such that $B\left(f_{x x^{\prime}}, x, x^{\prime}\right), B\left(f_{x^{\prime} x^{\prime \prime}}, x^{\prime}, x^{\prime \prime}\right)$ it is possible to compose them in some way to get $f_{x x^{\prime \prime}}$ such that $B\left(f_{x x^{\prime \prime}}, x, x^{\prime \prime}\right)$, then $R$ is indeed a preorder.

2000 Mathematics Subject Classification: 03E15, 54F15. 
The main known examples of universal analytic preorders are in fact notions of embeddability for various classes of algebraic or topological structures. The universality of embeddability for countable graphs is proved in [LR05] and various kinds of embeddability relations for coloured countable total orders are proved universal in [MR04] and [Ca]. Moreover, [LR05] proves that continuous embeddability for continua (compact connected metric spaces) is an analytic universal preorder. This result is strengthened in [MR04], where it is proved that universality already holds for continuous embeddability on dendrites (locally connected continua not containing simple closed curves), and in [Ca], where it is shown that this universality still holds upon restriction to dendrites of a special minimal kind.

The lack of examples of universal analytic preorders generated by requiring the existence of particular surjective functions was noted by U. B. Darji, who inquired - in private communications - about the universality of the relation on continua defined by letting $K \preceq K^{\prime}$ if and only if $K$ is a continuous image of $K^{\prime}$. This relation may be considered dual to continuous embeddability. However, there is a strong asymmetry from the start: while continuous embeddability is universal already on dendrites, no analogous result can hold for $\preceq$, since any two locally connected non-degenerate continua are continuous images of each other, so they form a single degree with respect to $\preceq$. Darji's question triggered the investigation of this paper, which may be considered as a contribution to the ongoing study of the interactions between descriptive set theory and continuum theory (for a survey of some recent results in this field, see [Mar]).

Since graphs are usually a first test for the study of relations on algebraic structures, Section 1 studies the relation of epimorphism for countable graphs and proves its $\boldsymbol{\Sigma}_{1}^{1}$-universality.

Section 2 answers Darji's question in the affirmative, proving that $\preceq$ is indeed a $\boldsymbol{\Sigma}_{1}^{1}$-universal preorder.

Though the relation $\preceq$ trivialises on dendrites, one gets more interesting preorders by restricting the class of continuous surjections allowed (see, for example, [CCP94]). In Section 3 it is proved that allowing only open continuous surjections one gets an analytic preorder $\preceq O$ that is universal already when restricted to the class of dendrites.

The study of classes of countable structures or of continua is performed in suitable spaces. A description of the space $X_{L}$ of structures with universe $\mathbb{N}$ for a given countable language $L$ can be found in [K95]. An account of the hyperspaces $K(X)$ of all compact subsets of a continuum $X$ and $C(X)$ of all subcontinua of $X$ is in [N92]. Since the Hilbert cube contains a homeomorphic copy of all compact metric spaces, $K\left([0,1]^{\mathbb{N}}\right)$ and $C\left([0,1]^{\mathbb{N}}\right)$ provide a suitable framework for their theory. Each of $X_{L}, K(X), C(X)$ is a Polish space. 
The results of this paper share a common basic idea and are proved using a technique developed in [FS89] to study universality for relations of isomorphism on classes of countable structures; the technique was also exploited in $[\mathrm{Ca}]$ to prove the universality of some embeddability relations. Here is a short account of what will be needed.

A graph is an irreflexive, symmetric binary relation. Let $\mathcal{L}$ be the language of graph theory, consisting of one binary relation symbol, besides equality. For every $n \in \mathbb{N}$, let $T Y_{n}$ be the set of quantifier free types for the first $n$ variables in $\mathcal{L}$ and let $T Y=\bigcup_{n \in \mathbb{N}} T Y_{n}$ (note that an empty 0-type is also considered here). Fix a bijection $e: \mathbb{N} \rightarrow T Y$ enumerating types such that, if $e(i) \in T Y_{n}$ and $e(j) \in T Y_{m}, n<m$, then $i<j$. Indeed, for each $n \in \mathbb{N}$ there are a finite number of $n$-types in $\mathcal{L}$. So each $i \in \mathbb{N}$ codes the type $e(i)$. For example, 0 codes the empty type. For $G$ a graph on $\mathbb{N}$ and $t \in \mathbb{N}^{<\omega}$ let $\tau_{G}(t) \in \mathbb{N}$ be the (code of the) quantifier free type of $t$ in $G$. Note that $\tau_{G}(t)$ determines $\tau_{G}(s)$ for all sequences $s$ whose values form a subset of the values taken by $t$. Also, $\tau_{G}(\emptyset)=0$ and the value $\tau_{G}(m)$ is the same for all graphs $G$ on $\mathbb{N}$ and all $m \in \mathbb{N}$, since each element of the graph equals itself and it is not adjacent to itself. Here and in what follows, 1-tuples from a set $A$ are identified with elements of $A$-which interpretation is meant will always be clear from the context.

If $G, H$ are graphs on $\mathbb{N}$ and $g: \mathbb{N} \rightarrow \mathbb{N}$, then $g$ is an embedding of $G$ into $H$ if and only if it is injective and $\forall a, b \in \mathbb{N}(a G b \Leftrightarrow g(a) H g(b))$. Note then the following. Let $G, H$ be graphs on $\mathbb{N}$ and let $g: \mathbb{N} \rightarrow \mathbb{N}$. Let $g^{\prime}: \mathbb{N}<\omega \rightarrow$ $\mathbb{N}^{<\omega}$ be defined from $g$ componentwise: $g^{\prime}\left(t_{0}, \ldots, t_{n-1}\right)=\left(g\left(t_{0}\right), \ldots, g\left(t_{n-1}\right)\right)$ for $\left(t_{0}, \ldots, t_{n-1}\right) \in \mathbb{N}^{n}$. Then $g$ is an embedding of $G$ into $H$ if and only if $\tau_{G}(t)=\tau_{H} g^{\prime}(t)$ for all $t \in \mathbb{N}^{<\omega}$.

The universality results in this paper are obtained by comparing, under Borel reducibility, analytic preorders with the relation of embeddability for graphs on $\mathbb{N}$ and using the following result of [LR05].

THEOREM. The relation of embeddability is a $\boldsymbol{\Sigma}_{1}^{1}$-universal preorder on the Polish space of graphs on $\mathbb{N}$.

Note also that if $\varrho$ is a $\Sigma_{1}^{1}$-universal preorder on some standard Borel space $X$, then so is $\varrho^{-1}$. Indeed, by the universality of $\varrho$, there is a Borel reduction $\varphi: X \rightarrow X$ of $\varrho^{-1}$ to $\varrho$; then $\varphi$ is also a reduction of $\left(\varrho^{-1}\right)^{-1}=\varrho$ to $\varrho^{-1}$.

1. Epimorphisms between graphs. Given a relational language $L$ and $L$-structures $M, M^{\prime}$, a function $g: M \rightarrow M^{\prime}$ is a homomorphism if and only if for any relation symbol $R$ of $L$, say of arity $n$,

$$
R^{M}\left(m_{1}, \ldots, m_{n}\right) \Rightarrow R^{M^{\prime}}\left(g\left(m_{1}\right), \ldots, g\left(m_{n}\right)\right), \quad \text { for all } m_{1}, \ldots, m_{n} \in M \text {. }
$$

An epimorphism is a surjective homomorphism. The universality of homomorphism between graphs on $\mathbb{N}$ is proven in [LR05]. 
TheOREM 1. There is a continuous function $\varphi: G \mapsto G^{*}$ from the class of graphs on $\mathbb{N}$ to itself such that $G$ embeds into $H$ if and only if there is an epimorphism of $H^{*}$ onto $G^{*}$. Consequently, the relation of being epimorphic image is a $\boldsymbol{\Sigma}_{1}^{1}$-universal preorder for graphs on $\mathbb{N}$.

Proof. Let $\left\{N_{t}\right\}_{t \in \mathbb{N}<\omega}$ be a partition of $\mathbb{N}$ into infinite sets. Within each $N_{t}$ fix distinct elements $a^{t}, c_{i}^{t}$ for $i \in \mathbb{N}$ so that $N_{t} \backslash\left\{a^{t}, c_{i}^{t}\right\}_{i \in \mathbb{N}}$ is still infinite. For each $t \in \mathbb{N}^{<\omega}$ and $n \in \mathbb{N}$ let $L_{t n}$ be a graph on $N_{t}$ with the properties:

- there are $n+2$ nodes $b_{1}^{t n}, \ldots, b_{n+2}^{t n} \notin\left\{a^{t}, c_{i}^{t}\right\}_{i \in \mathbb{N}}$ such that $B_{t n}=$ $\left\{a^{t}, b_{1}^{t n}, \ldots, b_{n+2}^{t n}\right\}$ forms a complete subgraph of $L_{t n}$ of order $n+3$;

- besides the other nodes of $B_{t n}, b_{n+2}^{t n}$ is adjacent to all $c_{i}^{t}$ and to a sequence of distinct nodes $d_{i}^{t n}$, for all $i \in \mathbb{N}$;

- all $c_{i}^{t}, d_{i}^{t n}$ are adjacent to each other (so $C_{t n}=\left\{c_{i}^{t}, d_{i}^{t n}\right\}_{i \in \mathbb{N}}$ forms an infinite complete subgraph of $L_{t n}$ );

- no other adjacency relation holds in $L_{t n}$;

- $N_{t}=B_{t n} \cup C_{t n}$ and this is a disjoint union.

Note that, as the cardinality of $B_{t n}$ depends on $n$, also the choice of the elements of $N_{t}$ forming the sequence of nodes $d_{i}^{t n}$ in $L_{t n}$ depends on $n$, while the elements $a^{t}, c_{i}^{t}$ can be fixed independently of $n$.

Let $G$ be a graph on $\mathbb{N}$. Then $G^{*}=\varphi(G)$ is the graph on $\mathbb{N}$ defined as follows:

- the adjacency relation on each $N_{t}$ is given by $L_{t \tau_{G}(t)}$;

- for each $t \in \mathbb{N}^{<\omega}$ and $i \in \mathbb{N}$ the nodes $c_{i}^{t}$ and $a^{t^{\wedge} i}$ are adjacent;

- no other adjacency relation holds in $G^{*}$.

So the map $\varphi: G \mapsto G^{*}$ is continuous. Note that each node $u$ of $G^{*}$ is either in some $B_{t \tau_{G}(t)}$ or in some $C_{t \tau_{G}(t)}$ (complete graphs of order $\tau_{G}(t)+3$ and $\aleph_{0}$ respectively), these cases being mutually exclusive. Since the adjacency relation $L_{t n}$ used on $N_{t}$ in the graph $G^{*}$ is the one for which $n=\tau_{G}(t)$, it is possible to simplify notation a little by writing $b_{i}^{t G}, d_{i}^{t G}, B_{t G}, C_{t G}$ for $b_{i}^{t \tau_{G}(t)}, d_{i}^{t \tau_{G}(t)}, B_{t \tau_{G}(t)}, C_{t \tau_{G}(t)}$, respectively.

To show that $\varphi$ is a reduction, fix graphs $G, H$ on $\mathbb{N}$.

Suppose $g: \mathbb{N} \rightarrow \mathbb{N}$ is an embedding of $G$ into $H$. We will define an epimorphism $h$ of $H^{*}$ onto $G^{*}$. Let $g^{\prime}: \mathbb{N}^{<\omega} \rightarrow \mathbb{N}^{<\omega}$ be defined from $g$ componentwise, so that $\tau_{G}(t)=\tau_{H} g^{\prime}(t)$ for all $t \in \mathbb{N}^{<\omega}$. Let $\Gamma$ be the subgraph of $G^{*}$ obtained by removing all $d_{i}^{t G}$ for all $t \in \mathbb{N}<\omega$ and $i \in \mathbb{N}$. Let $k$ be the embedding of $\Gamma$ into $H^{*}$ defined on each $N_{t} \backslash\left\{d_{i}^{t G}\right\}_{i \in \mathbb{N}}$ by:

- $k\left(a^{t}\right)=a^{g^{\prime}(t)}$;

- $k\left(b_{j}^{t G}\right)=b_{j}^{g^{\prime}(t) H}$ for $j \in\left\{1, \ldots, \tau_{G}(t)+2\right\}$;

- $k\left(c_{i}^{t}\right)=c_{g(i)}^{g^{\prime}(t)}$ for $i \in \mathbb{N}$.

Define $h$ on $\operatorname{im} k$ (the range of $k$ ) as the inverse of $k$. 
If $g$ is also surjective (and so an isomorphism), then the only nodes of $H^{*}$ not covered by im $k$ are those of the form $d_{j}^{r H}$ and one can extend $h$ to an isomorphism of $H^{*}$ onto $G^{*}$ by $h\left(d_{j}^{r H}\right)=d_{j}^{t G}$, where $g^{\prime}(t)=r$.

Otherwise, if $u$ is a node of $H^{*}$ not in $\operatorname{im} k$ there are three possibilities:

- $u=d_{j}^{r H}$ for some $r=r_{u} \in \operatorname{im} g^{\prime}, j \in \mathbb{N}$;

- $u=c_{j}^{r}$ for some $r=r_{u} \in \operatorname{im} g^{\prime}, j \notin \operatorname{im} g$;

- $u \in N_{r^{\wedge} j^{\wedge} s}$ for some $r=r_{u} \in \operatorname{im} g^{\prime}, j \notin \operatorname{im} g, s \in \mathbb{N}^{<\omega}$.

Note that this $r_{u} \in \operatorname{im} g^{\prime}$ is uniquely determined by $u$. So fix $r \in \operatorname{im} g^{\prime}$ and let $t$ be the unique preimage of $r$ under $g^{\prime}$. Let $\varrho: \mathbb{N} \rightarrow \operatorname{img}$ be a bijection (indeed, im $g$ is infinite). Define $h\left(d_{j}^{r H}\right)=d_{\varrho(j)}^{t G}$. Finally, for each $j \in \mathbb{N} \backslash \operatorname{im} g$, let $h\left(c_{j}^{r}\right)=d_{j}^{t G}$ and then extend the definition of $h$ to the subset $\bigcup_{s \in \mathbb{N}<\omega} N_{r^{\wedge} j^{\wedge} s}$ of $H^{*}$ by injecting it into the infinite complete graph $C_{t G}$, with the only requirement that $h\left(a^{r^{\wedge} j}\right) \neq d_{j}^{t G}$.

Conversely, let $h$ be an epimorphism of $H^{*}$ onto $G^{*}$. Note that if $K_{\alpha}$ is a complete subgraph of $H^{*}$ of order $\alpha \leq \aleph_{0}$, then $\left.h\right|_{K_{\alpha}}$ is an embedding of $K_{\alpha}$ onto a complete subgraph of order $\alpha$ of $G^{*}$. This implies that if $u \in B_{r H} \backslash\left\{b_{\tau_{H}(r)+2}^{r H}\right\}$, the degree of $h(u)$ in $G^{*}$ is at least $\tau_{H}(r)+2$, while if $u \in C_{r H} \cup\left\{b_{\tau_{H}(r)+2}^{r H}\right\}$, the degree of $h(u)$ is infinite. Moreover, $h\left(C_{r H}\right)$ is a subset of some $C_{t G} \cup\left\{b_{\tau_{G}(t)+2}^{t G}\right\}$ and $h\left(B_{r H}\right)$ is either a subset of some $C_{t G} \cup\left\{b_{\tau_{G}(t)+2}^{t G}\right\}$ or of some $B_{t G}$ with $\tau_{G}(t) \geq \tau_{H}(r)$ (in particular, length $(t) \geq \operatorname{length}(r)$; moreover, in this case, $\left.h\left(b_{\tau_{H}(r)+2}^{r H}\right)=b_{\tau_{G}(t)+2}^{t G}\right)$. Observe that $h\left(B_{\emptyset H}\right)=B_{\emptyset G}$ as otherwise $a^{\emptyset} \notin \operatorname{im} h$. So $h\left(b_{\tau_{H}(\emptyset)+2}^{\emptyset H}\right)=b_{\tau_{G}(\emptyset)+2}^{\emptyset G}$ and $h$ embeds $C_{\emptyset H}$ into $C_{\emptyset G}$.

In order for $a^{0}$ to be in the range of $h$ there must be some $j_{0} \in \mathbb{N}$ with $h\left(B_{j_{0} H}\right) \subseteq B_{0 G}$. Note that such a $j_{0}$ is unique, since if $j_{0}^{\prime}$ were a different one then $h\left(c_{j_{0}}^{\emptyset}\right)=h\left(c_{j_{0}^{\prime}}^{\emptyset}\right)=c_{0}^{\emptyset}$, contrary to the adjacency of $c_{j_{0}}^{\emptyset}, c_{j_{0}^{\prime}}^{\emptyset}$ in $H^{*}$. So actually $h\left(B_{j_{0} H}\right)=B_{0 G}$, with $h\left(a^{j_{0}}\right)=a^{0}$ and $h\left(b_{\tau_{H}\left(j_{0}\right)+2}^{j_{0} H}\right)=b_{\tau_{G}(0)+2}^{0 G}$, implying $\tau_{G}(0)=\tau_{H}\left(j_{0}\right)$. As a consequence, $h$ embeds $C_{j_{0} H}$ into $C_{0 G}$. Note also that if $r(0) \neq j_{0}$ and $t(0)=0$, then no point $u \in N_{r} \subseteq H^{*}$ can be sent by $h$ to some $v \in N_{t} \subseteq G^{*}$, since otherwise $a^{0}$ would be the image of a point from any path in $H^{*}$ from $c_{r(0)}^{\emptyset}$ to $u$, so $a^{0}$ would be the image of a vertex from some $B_{s H}$ or from some $C_{s H}$, with $s(0)=r(0)$ and $s \subseteq r$. This is impossible since either length $(s)=1$ and $s(0) \neq j_{0}$, or length $(s)>1$.

Inductively, suppose $h\left(B_{\left(j_{0}, \ldots, j_{n}\right) H}\right)=B_{(0, \ldots, n) G}$ (implying $\tau_{G}(0, \ldots, n)=$ $\left.\tau_{H}\left(j_{0}, \ldots, j_{n}\right)\right)$, with

$$
h\left(a^{\left(j_{0}, \ldots, j_{n}\right)}\right)=a^{(0, \ldots, n)}, \quad h\left(b_{\tau_{H}\left(j_{0}, \ldots, j_{n}\right)+2}^{\left(j_{0}, \ldots, j_{n}\right) H}\right)=b_{\tau_{G}(0, \ldots, n)+2}^{(0, \ldots, n) G}
$$


(so $h$ embeds $C_{\left(j_{0}, \ldots, j_{n}\right) H}$ into $\left.C_{(0, \ldots, n) G)}\right)$; suppose moreover that if $(0, \ldots, n)$ $\subseteq t$ and $\left(j_{0}, \ldots, j_{n}\right) \nsubseteq r$ then no point of $N_{r}$ is sent by $h$ to a point of $N_{t}$. Then in order for $a^{(0, \ldots, n+1)}$ to be in the range of $h$ there is a unique $j_{n+1} \in \mathbb{N}$ such that $h\left(B_{\left(j_{0}, \ldots, j_{n+1}\right) H}\right) \subseteq B_{(0, \ldots, n+1) G}$, with

$$
\begin{gathered}
h\left(c_{j_{n+1}}^{\left(j_{0}, \ldots, j_{n}\right)}\right)=c_{n+1}^{(0, \ldots, n)}, \quad h\left(a^{\left(j_{0}, \ldots, j_{n+1}\right)}\right)=a^{(0, \ldots, n+1)}, \\
h\left(b_{\tau_{H}\left(j_{0}, \ldots, j_{n+1}\right)+2}^{\left(j_{0}, \ldots, j_{n+1}\right) H}\right)=b_{\tau_{G}(0, \ldots, n+1)+2}^{(0, \ldots, n+1) G} .
\end{gathered}
$$

Thus, $h$ embeds $C_{\left(j_{0}, \ldots, j_{n+1}\right) H}$ into $C_{(0, \ldots, n+1) G}$. Since no $r \neq\left(j_{0}, \ldots, j_{n+1}\right)$ is such that $h\left(B_{r H}\right) \subseteq B_{(0, \ldots, n+1) G}$, we have $h\left(B_{\left(j_{0}, \ldots, j_{n+1}\right) H}\right)=B_{(0, \ldots, n+1) G}$ (entailing $\left.\tau_{G}(0, \ldots, n+1)=\tau_{H}\left(j_{0}, \ldots, j_{n+1}\right)\right)$. Moreover, let $(0, \ldots, n+1)$ $\subseteq t$ and $\left(j_{0}, \ldots, j_{n+1}\right) \nsubseteq r$. If $\left(j_{0}, \ldots, j_{n}\right) \nsubseteq r$, then no point of $N_{r}$ is sent by $h$ to a point of $N_{t}$ by inductive hypothesis. If $\left(j_{0}, \ldots, j_{n}, j\right) \subseteq r$ with $j \neq j_{n+1}$, then no point $u \in N_{r}$ can be sent by $h$ to a point of $N_{t}$, since otherwise the vertex $a^{(0, \ldots, n+1)}$ would be image of some point of $v \in N_{s}$ with $\left(j_{0}, \ldots, j_{n}, j\right) \subseteq s \subseteq r$, giving rise to three possible cases:

(i) $s=\left(j_{0}, \ldots, j_{n}, j\right), v \in B_{s H}$;

(ii) $\left(j_{0}, \ldots, j_{n}, j\right) \subset s, v \in B_{s H}$;

(iii) $v \in C_{s H}$.

Cases (ii) and (iii) are impossible since $B_{s H}$ (for case (ii)) or $C_{s H}$ (for case (iii)) cannot be embedded in $B_{(0, \ldots, n+1) G}$; case (i) is impossible, since otherwise $h\left(c_{j}^{\left(j_{0}, \ldots, j_{n}\right)}\right)=h\left(c_{j_{n+1}}^{\left(j_{0}, \ldots, j_{n}\right)}\right)=c_{n+1}^{(0, \ldots, n)}$, contrary to the adjacency of $c_{j}^{\left(j_{0}, \ldots, j_{n}\right)}, c_{j_{n+1}}^{\left(j_{0}, \ldots, j_{n}\right)}$ in $H^{*}$.

In this way, a sequence $\left(j_{0}, j_{1}, j_{2}, \ldots\right)$ is built such that $\tau_{G}(0,1, \ldots, n)=$ $\tau_{H}\left(j_{0}, j_{1}, \ldots, j_{n}\right)$ for all $n \in \mathbb{N}$, showing that $\mathbb{N} \rightarrow \mathbb{N}, i \mapsto j_{i}$, is an embedding of $G$ into $H$.

2. Continuous surjections between continua. Let $\preceq$ be the preorder on $C\left([0,1]^{\mathbb{N}}\right)$ defined by letting $K \preceq K^{\prime}$ if and only if there is a continuous surjection $K^{\prime} \rightarrow K$.

Lemma 2. The preorder $\preceq$ is analytic.

Proof. For $K, K^{\prime} \in C\left([0,1]^{\mathbb{N}}\right)$, the existence of a continuous surjection from $K^{\prime}$ onto $K$ can be expressed by requiring the existence of $f \in C\left([0,1]^{\mathbb{N}} \times\right.$ $\left.[0,1]^{\mathbb{N}}\right)$ such that if we let $\pi_{1}, \pi_{2}$ be the two projections from $[0,1]^{\mathbb{N}} \times[0,1]^{\mathbb{N}}$ onto its two factors, the following holds:

(i) $\forall x, y, y^{\prime} \in[0,1]^{\mathbb{N}}\left((x, y) \in f \wedge\left(x, y^{\prime}\right) \in f \Rightarrow y=y^{\prime}\right)$;

(ii) $\pi_{1}(f)=K^{\prime}$;

(iii) $\pi_{2}(f)=K$.

The listed conditions are Borel in $\left(K, K^{\prime}, f\right)$. 
This section is devoted to the proof of $\boldsymbol{\Sigma}_{1}^{1}$-universality of $\preceq$. The result of [MR04] shows that the $\boldsymbol{\Sigma}_{1}^{1}$-universality of continuous embeddability between continua holds already when restricted to the class of dendrites (and in fact, by [Ca], to a quite small subclass of them). Dendrites are fairly simple continua, in particular they are locally connected. So no analogous result can hold for the relation $\preceq$; indeed, any two locally connected non-degenerate continua are continuous images of each other (however, the situation is very different when considering restricted subclasses of continuous surjections, see [CCP94] and the next section). So to establish the $\boldsymbol{\Sigma}_{1}^{1}$-universality of $\preceq$ a very different class of continua must be employed. A key role in the construction will be indeed played by a Cook continuum $X$, as constructed in [Mać86]. Recall that a continuum is hereditarily non-divisible by points if and only if, for any subcontinuum $Y$ and $y \in Y$, the point $y$ does not separate $Y$. The important features of $X$ that will be used are:

- $X$ is a non-degenerate subcontinuum of $\mathbb{R}^{2}$;

- $X$ is hereditarily non-divisible by points;

- if $K$ is a subcontinuum of $X$ and $f: K \rightarrow X$ is a continuous function, then either $f$ is constant or $f$ is identity on $K$.

THEOREM 3. There is a continuous function $G \mapsto G^{*}$ assigning to each graph on $\mathbb{N}$ a subcontinuum of $[0,1]^{\mathbb{N}}$ in such a way that, given graphs $G, H$ on $\mathbb{N}$, there is an embedding of $G$ into $H$ if and only if $G^{*}$ is a continuous image of $H^{*}$. Consequently, $\preceq$ is a $\boldsymbol{\Sigma}_{1}^{1}$-universal preorder.

Proof. Let $\left\{X^{j}\right\}_{j \in \mathbb{N}}$ be a collection of subcontinua of $[0,1]^{2}$ homeomorphic to pairwise disjoint non-degenerate subcontinua of $X$ each containing the points $q=q_{\emptyset}=(0,0)$ and $p=p_{\emptyset}=(1,1)$. Let $\left\{N_{t}\right\}_{t \in \mathbb{N}<\omega}$ be a partition of $\mathbb{N}$ into two-element sets, with $N_{\emptyset}=\{0,1\}$. For ease of notation, if $N \subseteq N^{\prime} \subseteq \mathbb{N}$ think of $\mathbb{R}^{N}$ as naturally embedded in $\mathbb{R}^{N^{\prime}}$, that is, the $N^{\prime} \backslash N$ coordinates of a point of $\mathbb{R}^{N}$ are null. So a subset of $\mathbb{R}^{N}$ is also a subset of $\mathbb{R}^{N^{\prime}}$. When $N$ is finite and $x \in \mathbb{R}^{N}$ is written as $x=\left(x_{1}, \ldots, x_{\operatorname{card}(N)}\right)$, the listed coordinates correspond to indices in $N$, and it will be clear from the context which space $x$ is thought to be in.

For any $j \in \mathbb{N}$ and $t \in \mathbb{N}<\omega$, let $X_{t}^{j} \subseteq[0,1]^{N_{t}}$ be the continuum obtained as a copy of $X^{j}$ by increasingly renaming the coordinates so that they belong to $N_{t}$. Let $q_{t}, p_{t}$ be the points corresponding to $q, p$, respectively; these are the points with both coordinates 0 , respectively 1 , in the appropriate square.

Let $G$ be a graph on $\mathbb{N}$. We define $G^{*}=\overline{\bigcup_{n \in \mathbb{N}} G_{n}}$, the closure of an increasing union of continua $G_{n}$, which, in turn, are defined inductively.

To begin with, let $G_{0}=X_{\emptyset}^{G}$ where $X_{\emptyset}^{G}=X_{\emptyset}^{\tau_{G}(\emptyset)}=X_{\emptyset}^{0}$. Let $z_{\emptyset}^{G}=p_{\emptyset}$, the point with 1 at the $N_{\emptyset}$ coordinates and 0 elsewhere. This is the point where the construction will grow at the next step. 
The next step is to define

$$
G_{1}=G_{0} \cup \bigcup_{i_{0} \in \mathbb{N}} X_{i_{0}}^{G}, \quad \text { where } \quad X_{i_{0}}^{G}=\left\{z_{\emptyset}^{G}\right\} \times X_{i_{0}}^{\tau_{G}\left(i_{0}\right)} .
$$

Note that so far the construction does not really depend on $G$, as there are a unique 0 -type and a unique 1-type realisable by graphs. The construction will grow at the points $z_{i_{0}}^{G}=\left(z_{\emptyset}^{G}, p_{i_{0}}\right)$, having 1 at the $N_{\emptyset} \cup N_{i_{0}}$ coordinates and 0 elsewhere, for all $i_{0} \in \mathbb{N}$.

Next step:

$$
G_{2}=G_{1} \cup \bigcup_{\left(i_{0}, i_{1}\right) \in \mathbb{N}^{2}} X_{\left(i_{0}, i_{1}\right)}^{G}, \quad \text { where } \quad X_{\left(i_{0}, i_{1}\right)}^{G}=\left\{z_{i_{0}}^{G}\right\} \times X_{\left(i_{0}, i_{1}\right)}^{\tau_{G}\left(i_{0}, i_{1}\right)} .
$$

The construction will resume at points $z_{\left(i_{0}, i_{1}\right)}^{G}=\left(z_{i_{0}}^{G}, p_{\left(i_{0}, i_{1}\right)}\right)$, whose coordinates are 1 in $N_{\emptyset} \cup N_{i_{0}} \cup N_{\left(i_{0}, i_{1}\right)}$ and 0 elsewhere, for all $\left(i_{0}, i_{1}\right) \in \mathbb{N}^{2}$.

In general, assuming the construction performed up to level $n$, let

$$
G_{n+1}=G_{n} \cup \bigcup_{t \in \mathbb{N}^{n+1}} X_{t}^{G}, \quad \text { where } \quad X_{t}^{G}=\left\{z_{\left.t\right|_{n}}^{G}\right\} \times X_{t}^{\tau_{G}(t)} ;
$$

set also $z_{t}^{G}=\left(z_{\left.t\right|_{n}}^{G}, p_{t}\right)$; these points have exactly $2(n+2)$ coordinates equal to 1 and are the points where the construction will continue.

Note that $X_{\emptyset}^{G} \backslash\left\{z_{\emptyset}^{G}\right\}$ is open in $G^{*}$; similarly, for each $t \in \mathbb{N}^{<\omega}$ and $i \in \mathbb{N}$, the sets $X_{t^{\wedge} i}^{G} \backslash\left\{z_{t}^{G}, z_{t^{\wedge} i}^{G}\right\}$ are open in $G^{*}$. The remainder $R_{G}=$ $G^{*} \backslash \bigcup_{n \in \mathbb{N}} G_{n}$ is homeomorphic to the Baire space via $\xi \mapsto z_{\xi}^{G}=\lim _{n \rightarrow \infty} z_{\left.\xi\right|_{n}}^{G}$. Also, $\bar{R}_{G}=R_{G} \cup\left\{z_{t}^{G}\right\}_{t \in \mathbb{N}<\omega}$ is homeomorphic to the Cantor space, being compact, perfect, and zero-dimensional.

Note that the upper indices $G$ in $z_{t}^{G}, z_{\xi}^{G}$ are unnecessary, since these points are fixed in the Hilbert cube and are the same for all $G$. However, this notation will help to identify the space $G^{*}$ where these points are thought of as elements.

Let $G, H$ be graphs on $\mathbb{N}$.

Suppose first that $g: \mathbb{N} \rightarrow \mathbb{N}$ is an embedding of $G$ into $H$. We wish to find a continuous surjection $h: H^{*} \rightarrow G^{*}$. Define $g^{\prime}: \mathbb{N}^{<\omega} \cup \mathbb{N}^{\mathbb{N}} \rightarrow \mathbb{N}^{<\omega} \cup \mathbb{N}^{\mathbb{N}}$ from $g$ componentwise. Then $\tau_{G}(t)=\tau_{H} g^{\prime}(t)$ for all $t \in \mathbb{N}<\omega$. The first step is to define piecewise a continuous injection $k: G^{*} \rightarrow H^{*}$. For $t \in \mathbb{N}^{<\omega}$, let $\left.k\right|_{X_{t}^{G}}$ map homeomorphically onto $X_{g^{\prime}(t)}^{H}$. This homeomorphism is unique by the properties of $X$. Note that $k\left(z_{t}^{G}\right)=z_{g^{\prime}(t)}^{H}$, so the function is defined consistently on each $X_{t}^{G} \cap X_{t^{-} i}^{G}=\left\{z_{t}^{G}\right\}$. Finally, set $k\left(z_{\xi}^{G}\right)=z_{g^{\prime}(\xi)}^{H}$.

Define $h$ on im $k$ as the inverse of $k$. Note that if $y \in H^{*} \backslash\left(\operatorname{im} k \cup R_{H}\right)$, then $y \in X_{s}^{H}$ for some $s \in \mathbb{N}<\omega$, where $s \notin \operatorname{im} g^{\prime}$. Let $t^{\prime}$ be the largest initial segment of $s$ such that $t^{\prime} \in \operatorname{im} g^{\prime}$ and suppose $t^{\prime}=g^{\prime}(t)$. Set $h(y)=z_{t}^{G}$ and extend the definition to $R_{H} \backslash \operatorname{im} k$ by continuity: $h\left(z_{t^{\prime} \zeta}^{H}\right)=z_{t}^{G}$ for $\zeta \in \mathbb{N}^{\mathbb{N}}$. 
This completes the definition of $h: H^{*} \rightarrow G^{*}$, which is surjective since its range includes $\operatorname{dom} k=G^{*}$.

Moreover, $h$ is continuous. Indeed, let $y_{n}$ be a sequence in $H^{*}$ converging to $y$. If almost all terms belong to $\operatorname{im} k$, then $\lim _{n \rightarrow \infty} h\left(y_{n}\right)=h(y)$, as $k$ is a homeomorphism on its range. So it may be assumed that $y_{n} \in H^{*} \backslash \operatorname{im} k$ for all $n \in \mathbb{N}$. For each $n \in \mathbb{N}$ there exist a unique finite sequence $s_{n} \in \operatorname{im} g^{\prime}$ and a natural number $i_{n} \notin \operatorname{im} g$ such that

$$
y_{n} \in \overline{\bigcup_{s \supseteq s_{n} i_{n}} X_{s}^{H}} .
$$

If $t_{n}$ is the unique preimage of $s_{n}$ under $g^{\prime}$, then $h\left(y_{n}\right)=h\left(z_{s_{n}}^{H}\right)=z_{t_{n}}^{G}$. If almost all $y_{n}$ share the same $s_{n}=\bar{s}$ and the same $i_{n}=\bar{i}$, then

$$
y \in \overline{\bigcup_{s \supseteq \bar{s}^{\wedge} \bar{i}} X_{s}^{H}},
$$

so $\lim _{n \rightarrow \infty} h\left(y_{n}\right)=h(y)=h\left(z_{\bar{s}}^{H}\right)$. If this is not the case but there is $\bar{s}$ such that $\bar{s}=s_{n}$ for infinitely many $n$ then $y=z_{\bar{s}}^{H}$, so eventually $s_{n}=\bar{s}$, proving $\lim _{n \rightarrow \infty} h\left(y_{n}\right)=h\left(z_{\bar{s}}^{H}\right)=h(y)$. If for each $s \in \operatorname{im} g^{\prime}$ there are only finitely many $n$ with $s_{n}=s$, then $\lim _{n \rightarrow \infty} d\left(y_{n}, z_{s_{n}}^{H}\right)=0$, so $y=\lim _{n \rightarrow \infty} z_{s_{n}}^{H}$ and $\lim _{n \rightarrow \infty} h\left(y_{n}\right)=\lim _{n \rightarrow \infty} h\left(z_{s_{n}}^{H}\right)=h(y)$ as $z_{s_{n}}^{H}, y \in \operatorname{im} k$.

Conversely, suppose $h: H^{*} \rightarrow G^{*}$ is a continuous surjection. We will define $g: \mathbb{N} \rightarrow \mathbb{N}$ embedding $G$ into $H$.

Claim. For $t^{\prime} \in \mathbb{N}^{<\omega}$, if $h\left(X_{t^{\prime}}^{H}\right)$ is non-degenerate then it equals $X_{t}^{G}$ for some $t \in \mathbb{N}<\omega$ with $\tau_{G}(t)=\tau_{H}\left(t^{\prime}\right)$, the restriction of $h$ to $X_{t^{\prime}}^{H}$ being the unique homeomorphism $X_{t^{\prime}}^{H} \rightarrow X_{t}^{G}$.

Proof of claim. Note that, under the assumption, it is enough to show that $h\left(X_{t^{\prime}}^{H}\right) \subseteq X_{t}^{G}$ for some $t$. Deny this.

Consider first the case $t^{\prime}=\emptyset$. Since $\bar{R}_{G}$ is totally disconnected, using the boundary bumping theorem there must be a non-degenerate subcontinuum $C$ of $h\left(X_{\emptyset}^{H}\right) \cap X_{t}^{G}$ for some $t \neq \emptyset$. Let $s$ be the restriction of $t$ to length $(t)-1$. Let $c \in C \backslash\left\{z_{s}^{G}, z_{t}^{G}\right\}$ and $c^{\prime} \in X_{\emptyset}^{H}$ with $h\left(c^{\prime}\right)=c$; let $U$ be an open neighbourhood of $c$ in $G^{*}$ with $U \subseteq X_{t}^{G}$ (for example $U=X_{t}^{G} \backslash\left\{z_{s}^{G}, z_{t}^{G}\right\}$ ) and set $V=\left(\left.h\right|_{X_{\emptyset}^{H}}\right)^{-1}(U)$. If $V=X_{\emptyset}^{H}$ then $h\left(X_{\emptyset}^{H}\right)$ is a non-degenerate subcontinuum of $X_{t}^{G}$ (and actually a contradiction is reached anyway, as $\left.\tau_{G}(t) \neq \tau_{H}(\emptyset)\right)$. If $V$ is a proper subset of $X_{\emptyset}^{H}$, by the boundary bumping theorem there is a continuum $D \subseteq \bar{V}$ such that $c^{\prime} \in D$ and $D$ meets the boundary of $V$ in some point $c^{\prime \prime}$. Then $h(D)$ is a non-degenerate subcontinuum of $X_{t}^{G}$ since $h\left(c^{\prime}\right) \neq h\left(c^{\prime \prime}\right)$, and a contradiction follows. 
Assume now $t^{\prime} \neq \emptyset$. Suppose

$$
h\left(X_{t^{\prime}}^{H}\right) \nsubseteq \overline{\bigcup_{\tau_{G}(t)=\tau_{H}\left(t^{\prime}\right)} X_{t}^{G}} .
$$

Then there are $t \in \mathbb{N}<\omega$ with $\tau_{G}(t) \neq \tau_{H}\left(t^{\prime}\right)$ and a non-degenerate subcontinuum $C$ of $h\left(X_{t^{\prime}}^{H}\right) \cap X_{t}^{G}$. As above, let $c \in C \backslash\left\{z_{\left.t\right|_{\text {length }(t)-1} ^{G}}, z_{t}^{G}\right\}\left(c \in C \backslash\left\{z_{\emptyset}^{G}\right\}\right.$ if $t=\emptyset), c^{\prime} \in X_{t^{\prime}}^{H} \cap h^{-1}(\{c\})$ and $U$ be an open neighbourhood of $c$ in $G^{*}$ with $U \subseteq X_{t}^{G}$. If $V=\left(\left.h\right|_{X_{t^{\prime}}^{H}}\right)^{-1}(U)$ then, as above, a contradiction is reached both from $V=X_{t^{\prime}}^{H}$ and from $V \subset X_{t^{\prime}}^{H}$. Thus it follows that

$$
h\left(X_{t^{\prime}}^{H}\right) \subseteq \bigcup_{\tau_{G}(t)=\tau_{H}\left(t^{\prime}\right)} X_{t}^{G} .
$$

Since $h\left(X_{t^{\prime}}^{H}\right)$ is connected, there must exist $s \in \mathbb{N}<\omega$ and $A \subseteq \mathbb{N}$ such that $h\left(X_{t^{\prime}}^{H}\right) \subseteq \bigcup_{n \in A} X_{s^{\urcorner} n}^{G}$, where $\tau_{G}\left(s^{\frown} n\right)=\tau_{H}\left(t^{\prime}\right)$ for all $n \in A$ and $A$ is minimal, namely

$$
A=\left\{n \in \mathbb{N} \mid h\left(X_{t^{\prime}}^{H}\right) \cap X_{s^{\wedge} n}^{G} \nsubseteq\left\{z_{s}^{G}\right\}\right\} .
$$

It remains to show that $A$ is a singleton. Deny. Then $C_{n}=h\left(X_{t^{\prime}}^{H}\right) \cap X_{s^{\frown} n}^{G}$ is a non-degenerate continuum containing $z_{s}^{G}$, for all $n \in A$. Let $k=\left.h\right|_{X_{t^{\prime}}^{H}}$ and set $V_{n}=k^{-1}\left(C_{n} \backslash\left\{z_{s}^{G}\right\}\right)$. All $V_{n}$ are proper, open, non-empty subsets of $X_{t^{\prime}}^{H}$. Moreover they are pairwise disjoint. Let $p_{n} \in V_{n}$ and $K_{n}$ be a subcontinuum of $\bar{V}_{n}$ meeting the boundary of $V_{n}$ at some point $y$, with $p_{n} \in K_{n}$. Note that $k(y)=z_{s}^{G}$. Then $\left.k\right|_{K_{n}}$ is a non-constant continuous

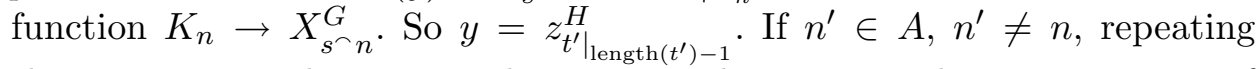
the argument within $V_{n^{\prime}}$ produces a non-degenerate subcontinuum $K_{n^{\prime}}$ of $X_{t^{\prime}}^{H}$ such that $K_{n} \cap K_{n^{\prime}}=\left\{\left.z_{t^{\prime}}^{H}\right|_{\text {length }\left(t^{\prime}\right)-1}\right\}$, contradicting hereditary nondivisibility by points of $X_{t^{\prime}}^{H}$.

Claim. $z_{\emptyset}^{G} \in h\left(X_{\emptyset}^{H}\right)$.

Proof of claim. Deny. By the above claim there is a point $\gamma \in G^{*} \backslash\left\{z_{\emptyset}^{G}\right\}$ such that $h\left(X_{\emptyset}^{H}\right)=\{\gamma\}$. If $\gamma \notin\left\{z_{t}^{G}\right\}_{t \in \mathbb{N}<\omega}$, then by induction on the length of $s$ it follows that $h\left(X_{s}^{H}\right)=\{\gamma\}$ for all $s \in \mathbb{N}^{<\omega}$. Indeed, assuming $h\left(X_{s}^{H}\right)=$ $\{\gamma\}$, from $h\left(z_{s}^{H}\right)=\gamma$ it follows by the previous claim that $h\left(X_{s^{\frown} n}^{H}\right)=\{\gamma\}$ for all $n \in \mathbb{N}$. Consequently, $h\left(H^{*}\right)=\{\gamma\}$. If $\gamma=z_{t}^{G}$ for some $t \in \mathbb{N}^{<\omega} \backslash\{\emptyset\}$, again by induction using the above claim it follows that each $h\left(X_{s}^{H}\right)$ is contained in some $X_{t_{s}}^{G}$, where $t \subseteq t_{s}$, thus $h$ is not surjective, since the points of $X_{\emptyset}^{G}$ are not in the range.

Let $\iota=(0,1,2, \ldots) \in \mathbb{N}^{\mathbb{N}}$. Using both claims one sees inductively that $h\left(H_{n}\right) \subseteq G_{n}$, so no point of $\bigcup_{n \in \mathbb{N}} H_{n}$ can be mapped by $h$ to $z_{\iota}^{G}$. So let $g \in \mathbb{N}^{\mathbb{N}}$ be such that $h\left(z_{g}^{H}\right)=z_{\iota}^{G}$. By the claims, either $h\left(X_{g(0)}^{H}\right)=\left\{z_{\emptyset}^{G}\right\}$ or 
$\left.h\right|_{X_{g(0)}^{H}}$ is the unique homeomorphism $X_{g(0)}^{H} \rightarrow X_{i_{0}}^{G}$ for some $i_{0} \in \mathbb{N}$. If the first alternative held, the first claim and induction would yield $h\left(X_{\left.g\right|_{n}}^{H}\right)=$ $\left\{z_{\emptyset}^{G}\right\}$ for all $n \geq 1$, contradicting $h\left(z_{g}^{H}\right)=z_{\iota}^{G}$. From the second alternative, again by induction using the first claim,

$$
\forall n \geq 1 \exists t_{n} \in \mathbb{N}^{\geq 1}\left(t_{n}(0)=i_{0} \wedge h\left(X_{\left.g\right|_{n}}^{H}\right) \subseteq X_{t_{n}}^{G}\right) .
$$

So in order that $h\left(z_{g}^{H}\right)=z_{\iota}^{G}$, we must have $i_{0}=0$. Suppose that $h\left(X_{\left.g\right|_{n}}^{H}\right)=$ $X_{(0,1, \ldots, n-1)}^{G}$ for some $n \geq 1$. Then either $h\left(X_{\left.g\right|_{n+1}}^{H}\right)=\left\{z_{(0, \ldots, n-1)}^{G}\right\}$ or there is $i_{n}$ such that $h\left(X_{\left.g\right|_{n+1}}^{H}\right)=X_{\left(0,1, \ldots, n-1, i_{n}\right)}^{G}$. The only possibility consistent with $h\left(z_{g}^{H}\right)=z_{\iota}^{G}$ is $h\left(X_{\left.g\right|_{n+1}}^{H}\right)=X_{(0, \ldots, n-1, n)}^{G}$. So $h\left(X_{\left.g\right|_{n}}^{H}\right)=X_{(0,1, \ldots, n-1)}^{G}$ for all $n \geq 1$, yielding $\tau_{G}(0,1, \ldots, n-1)=\tau_{H}(g(0), \ldots, g(n-1))$. The map $g: \mathbb{N} \rightarrow \mathbb{N}$ is thus an embedding of $G$ into $H$.

A continuous function $f: X \rightarrow Y$ between continua is said to be monotone if the preimage of each point of the range is connected (equivalently, the preimage of each subcontinuum of the range is a subcontinuum of $X$ ); it is weakly confluent if each subcontinuum of $Y$ is the image of a subcontinuum of $X$; and it is an r-mapping if it has a continuous right inverse $g: Y \rightarrow X$. Every motonone surjection between continua is weakly confluent. Under the hypothesis that $G$ embeds into $H$, the map $h: H^{*} \rightarrow G^{*}$ built in the proof of Theorem 3 is actually a monotone r-mapping ( $k$ is its right inverse). This remark establishes the following.

Corollary 4. The relation $\preceq_{M}$ on $C\left([0,1]^{\mathbb{N}}\right)$ defined by letting $K \preceq_{M}$ $K^{\prime}$ if and only if there is a monotone surjection $K^{\prime} \rightarrow K$ is a $\boldsymbol{\Sigma}_{1}^{1}$-universal preorder. Similarly for the relation $\preceq_{R}$ defined by $K \preceq_{R} K^{\prime}$ if and only if there is an r-mapping $K^{\prime} \rightarrow K$. The same holds for any analytic preorder $\theta$ on $C\left([0,1]^{\mathbb{N}}\right)$ with $\preceq_{M} \cap \preceq_{R} \subseteq \theta \subseteq \preceq$. For example, let $K \preceq_{W} K^{\prime}$ if and only if there is a weakly confluent surjection $K^{\prime} \rightarrow K$. Then $\preceq_{W}$ is a $\boldsymbol{\Sigma}_{1}^{1}$-universal preorder.

Proof. It remains to show that $\preceq_{M}, \preceq_{R}, \preceq_{W}$ are analytic.

For $\preceq_{M}$, add to the conditions of the proof of Lemma 2 the requirement

$$
\left(\mathrm{iv}_{m}\right) \forall y \in[0,1]^{\mathbb{N}} \pi_{1}\left(f \cap\left([0,1]^{\mathbb{N}} \times\{y\}\right)\right) \in C\left([0,1]^{\mathbb{N}}\right) \cup\{\emptyset\}
$$

or equivalently the condition

$$
\left(\mathrm{iv}_{m}^{\prime}\right) \forall y \in[0,1]^{\mathbb{N}} f \cap\left([0,1]^{\mathbb{N}} \times\{y\}\right) \in C\left(\left([0,1]^{\mathbb{N}}\right)^{2}\right) \cup\{\emptyset\}
$$

and recall that intersection is a Baire class 1 operation in $K\left([0,1]^{\mathbb{N}}\right)$ and for any continuum $X, C(X)$ is closed in $K(X)$.

For $\preceq_{R}$, the additional requirement is

$$
\left(\mathrm{iv}_{r}\right) \exists g \in C\left(\left([0,1]^{\mathbb{N}}\right)^{2}\right)\left(g: K \rightarrow K^{\prime} \wedge g^{-1} \subseteq f\right) .
$$


For $\preceq_{W}$, note that for a continuous surjection $f: K^{\prime} \rightarrow K$ to be weakly confluent it is enough that there exists a countable dense subset $\left\{C_{n}\right\}_{n \in \mathbb{N}}$ of $C(K)$ such that for all $n$ there is $C_{n}^{\prime} \in C\left(K^{\prime}\right)$ with $f\left(C_{n}^{\prime}\right)=C_{n}$. Indeed, fix then $C \in C(K)$ and let $C_{n_{k}}$ converge to $C$. Then a subsequence of $C_{n_{k}}^{\prime}$ converges to some continuum $C^{\prime} \subseteq K^{\prime}$ with $f\left(C^{\prime}\right)=C$. Thus, the characterisation of $\preceq_{W}$ is obtained by adding the following requirement to those of Lemma 2:

$$
\begin{gathered}
\left(\operatorname{iv}_{w}\right) \exists\left(C_{n}\right) \in\left(C\left([0,1]^{\mathbb{N}}\right)\right)^{\mathbb{N}}\left(\left\{C_{n}\right\}_{n \in \mathbb{N}} \text { is dense in } C(K) \wedge \forall n \in \mathbb{N} \exists C^{\prime} \in\right. \\
\left.C\left([0,1]^{\mathbb{N}}\right)\left(C^{\prime} \subseteq K^{\prime} \wedge f\left(C^{\prime}\right)=C_{n}\right)\right) .
\end{gathered}
$$

Now observe that $\left\{C_{n}\right\}_{n \in \mathbb{N}}$ dense in $C(K)$ means $\forall n \in \mathbb{N}\left(C_{n} \subseteq K\right) \wedge$ $\forall \varepsilon \in \mathbb{Q}^{+} \forall L \in C\left([0,1]^{\mathbb{N}}\right)\left(L \subseteq K \Rightarrow \exists n \in \mathbb{N}\left(d_{\mathrm{H}}\left(C_{n}, L\right)<\varepsilon\right)\right)\left(d_{\mathrm{H}}\right.$ being the Hausdorff metric on $\left.C\left([0,1]^{\mathbb{N}}\right)\right)$ while $f\left(C^{\prime}\right)=C_{n}$ means $\pi_{2}\left(f \cap\left(C^{\prime} \times\right.\right.$ $\left.\left.[0,1]^{\mathbb{N}}\right)\right)=C_{n}$ and both are Borel conditions.

REMARKS. 1. Similarly to what is done above, if $F$ is any class of continuous functions between continua containing all identities and closed under composition, one may define the relation $K \preceq_{F} K^{\prime}$ if and only if there is $f \in F$ mapping $K^{\prime}$ onto $K$ and, in case $\preceq_{F}$ is analytic, ask if it is a $\boldsymbol{\Sigma}_{1}^{1}$-universal preorder on $C\left([0,1]^{\mathbb{N}}\right)$. For the class $O$ of open functions, a much stronger result will be established in the next section.

2. The construction in the proof of Theorem 3 builds continua in the Hilbert cube. Indeed, it was notationally convenient to perform each step in the construction using countably many brand new coordinates. However, with some care, the same arguments can be developed in the square $[0,1]^{2}$.

To begin with, for every $t \in \mathbb{N}<\omega$ fix a 2-cell $W_{t} \subseteq[0,1]^{2}$ and distinct points $u_{t}, w_{t}$ in the manifold boundary of $W_{t}$ with the following properties:

- $W_{t} \cap W_{t^{\curvearrowright} n}=W_{t^{\curvearrowright} n} \cap W_{t^{\curvearrowright} m}=\left\{w_{t}\right\}=\left\{u_{t^{`} n}\right\}$ for $n \neq m$, while all other intersections $W_{t} \cap W_{s}$ with $t \neq s$ are empty;

- $\operatorname{diam}\left(W_{t^{\wedge} n}\right) \leq 2^{-n-1} \operatorname{diam}\left(W_{t}\right)$;

- let $w_{\xi}=\lim _{n \rightarrow \infty} w_{\left.\xi\right|_{n}}$ for $\xi \in \mathbb{N}^{\mathbb{N}}$; then $w_{\xi} \notin \bigcup_{t \in \mathbb{N}<\omega} W_{t}$ and $\xi \neq \xi^{\prime} \Rightarrow$ $w_{\xi} \neq w_{\xi^{\prime}}$.

For each $t \in \mathbb{N}<\omega$ and $j \in \mathbb{N}$ fix a continuum $Y_{t}^{j} \subseteq W_{t}$ homeomorphic to $X^{j}$ from the proof of Theorem 3 such that $u_{t}, w_{t} \in Y_{t}^{j}$. Given a graph $G$ on $\mathbb{N}$, let $G^{\prime}=\overline{\bigcup_{t \in \mathbb{N}<\omega} Y_{t}^{\tau_{G}(t)}}$. Then $G^{\prime}$ is homeomorphic to $G^{*}$ of the proof. The argument of the proof can then be repeated in the unit square and the results in this section hold as well for $C\left([0,1]^{2}\right)$.

3. Open continuous surjections between dendrites. Let $X$ be a continuum. If $p \in X$ denote by $\operatorname{ord}(p, X)$ the order of $p$ in $X$, that is, the smallest cardinal $\kappa$ such that there is an open neighbourhood basis 
of $p$ in $X$ whose members have boundary of cardinality at most $\kappa$. Let $R(X)=\{p \in X \mid \operatorname{ord}(p, X) \geq 3\}$ be the set of branching or ramification points of $X$ and $E(X)=\{p \in X \mid \operatorname{ord}(p, X)=1\}$ be the set of end points of $X$. An $\operatorname{arc} A \subseteq X$ with end points $a, b$ is a free $\operatorname{arc}$ in $X$ if $A \backslash\{a, b\}$ is open in $X$. In this case, $A \backslash\{a, b\}$ is an open free arc.

Using a definition from [Me67], for $i \in\{3,4,5,6\}$ let $D_{i}$ be the unique (up to homeomorphism) dendrite satisfying:

- if $p \in R\left(D_{i}\right)$, then $\operatorname{ord}\left(p, D_{i}\right)=i$;

- if $A \subseteq D_{i}$ is an arc, then $A \cap R\left(D_{i}\right) \neq \emptyset$.

The set $E\left(D_{i}\right)$ is dense in $D_{i}$, for each $i$. Fix distinct end points $x, y$ of $D_{6}$ and continuous open surjections $\varphi_{i}: D_{i+1} \rightarrow D_{i}$ for $i \in\{3,4,5\}$, whose existence is granted by [Ch80]. Let

$$
\begin{array}{lll}
x^{\prime}=\varphi_{5}(x), & x^{\prime \prime}=\varphi_{4} \varphi_{5}(x), & x^{\prime \prime \prime}=\varphi_{3} \varphi_{4} \varphi_{5}(x), \\
y^{\prime}=\varphi_{5}(y), & y^{\prime \prime}=\varphi_{4} \varphi_{5}(y), & y^{\prime \prime \prime}=\varphi_{3} \varphi_{4} \varphi_{5}(y) .
\end{array}
$$

Call $x\left(x^{\prime}, x^{\prime \prime}, x^{\prime \prime \prime}\right.$, respectively) the first special point of $D_{6}\left(D_{5}, D_{4}, D_{3}\right.$ respectively), and $y\left(y^{\prime}, y^{\prime \prime}, y^{\prime \prime \prime}\right.$, respectively) the second special point of $D_{6}$ ( $D_{5}, D_{4}, D_{3}$, respectively). Since end points are preserved by open continuous functions ([CCP94, Corollary 6.4]), special points are end points.

A string is a planar dendrite of the form $X=\bigcup_{n \in \mathbb{N}}\left(X_{n} \cup \alpha_{n}\right) \cup \alpha$ where:

$\left(1_{s}\right) \alpha$ is a free arc in $X$ with end points $p, q$ ( $q$ being called the final point and $\alpha$ the final arc of $X$ );

$\left(2_{s}\right)$ the $X_{n}$ are pairwise disjoint dendrites, with

$$
\alpha \cap X_{n}=\emptyset, \quad \lim _{n \rightarrow \infty} X_{n}=\{p\} ;
$$

$\left(3_{s}\right)$ the $\alpha_{n}$ are pairwise disjoint free $\operatorname{arcs}$ in $X$ with end points $b_{n}, a_{n+1}$, and with

$$
\begin{gathered}
\alpha \cap \alpha_{n}=\emptyset, \quad \lim _{n \rightarrow \infty} \alpha_{n}=\{p\}, \\
X_{m} \cap \alpha_{n}= \begin{cases}\emptyset & \text { if } n \neq m \neq n+1, \\
\left\{b_{n}\right\} & \text { if } m=n, \\
\left\{a_{n+1}\right\} & \text { if } m=n+1 ;\end{cases}
\end{gathered}
$$

(4s) $X_{n}$ is homeomorphic to

$$
\begin{cases}D_{6} & \text { if } n=0 \\ D_{4} & \text { if } n>0\end{cases}
$$

(5s) $a_{n}, b_{n}$ are the first and second special points of $X_{n}$.

Note that this definition of a string differs from that of [CCP94] by the presence of a final arc $\alpha$ instead of just a final point. 
A tame line is a planar dendrite of the form

$$
X=\alpha^{-} \cup \bigcup_{n \in \mathbb{Z}}\left(X_{n} \cup \alpha_{n}\right) \cup \alpha^{+},
$$

where:

$\left(1_{t}\right) \alpha^{-}, \alpha^{+}$are disjoint free arcs in $X$, with end points $q^{-}, p^{-}$and $p^{+}, q^{+}$ respectively $\left(q^{-}\right.$will be called the initial point of $X, q^{+}$the final point of $X, \alpha^{-}, \alpha^{+}$the initial and final arcs of $X$, respectively);

$\left(2_{t}\right)$ the $X_{n}$ are pairwise disjoint dendrites, with

$$
\alpha^{ \pm} \cap X_{n}=\emptyset, \quad \lim _{n \rightarrow \infty} X_{ \pm n}=\left\{p^{ \pm}\right\} ;
$$

$\left(3_{t}\right)$ the $\alpha_{n}$ are pairwise disjoint free $\operatorname{arcs}$ in $X$ with end points $b_{n}, a_{n+1}$, and with

$$
\begin{gathered}
\alpha^{ \pm} \cap \alpha_{n}=\emptyset, \quad \lim _{n \rightarrow \infty} \alpha_{ \pm n}=\left\{p^{ \pm}\right\}, \\
X_{m} \cap \alpha_{n}= \begin{cases}\emptyset & \text { if } n \neq m \neq n+1, \\
\left\{b_{n}\right\} & \text { if } m=n, \\
\left\{a_{n+1}\right\} & \text { if } m=n+1 ;\end{cases}
\end{gathered}
$$

$\left(4_{t}\right) X_{n}$ is homeomorphic to

$$
\begin{cases}D_{3} & \text { if } n<0 \\ D_{4} & \text { if } n \geq 0\end{cases}
$$

$\left(5_{t}\right) a_{n}, b_{n}$ are the first and second special points of $X_{n}$.

Let $\varrho$ be a prime number. A $\varrho$-line is defined as a tame line, except that condition $\left(4_{t}\right)$ is replaced by

$\left(4_{l}\right) X_{n}$ is homeomorphic to

$$
\begin{cases}D_{3} & \text { if } n<0 \\ D_{5} & \text { if } n \geq 0 \text { is a multiple of } \varrho \\ D_{4} & \text { otherwise. }\end{cases}
$$

In each of these cases, the continuum $X_{n}$ will be called the $n$th bead of the string or line, while $\alpha_{n}$ the $n$th bridge.

For $X, X^{\prime} \in C\left([0,1]^{\mathbb{N}}\right)$ let $X \preceq_{O} X^{\prime}$ if and only if there is an open continuous surjection from $X^{\prime}$ onto $X$.

Lemma 5. The preorder $\preceq_{O}$ is analytic.

Proof. By [E35], a continuous surjection $f$ between continua $X^{\prime}, X$ is open if and only if the function $F: X \rightarrow K\left(X^{\prime}\right), y \mapsto f^{-1}(\{y\})$, is continuous. So, given $X, X^{\prime} \in C\left([0,1]^{\mathbb{N}}\right), X \preceq_{O} X^{\prime}$ if and only if there exist $f \in C\left([0,1]^{\mathbb{N}} \times[0,1]^{\mathbb{N}}\right)$ and $F \in C\left([0,1]^{\mathbb{N}} \times K\left([0,1]^{\mathbb{N}}\right)\right)$ such that: 
(i) $f, F$ are graphs of functions;

(ii) $\pi_{1}(f)=X^{\prime}, \pi_{2}(f)=X, \pi_{1}(F)=X$;

(iii) $\forall y \in[0,1]^{\mathbb{N}}\left(\pi_{1}\left(f \cap\left([0,1]^{\mathbb{N}} \times\{y\}\right)\right)=\pi_{2}\left(F \cap\left(\{y\} \times K\left([0,1]^{\mathbb{N}}\right)\right)\right)\right)$.

Recall that the class $\mathcal{D}$ of dendrites is a Borel subset of $C\left([0,1]^{\mathbb{N}}\right)$ (namely, it is $\Pi_{3}^{0}$-complete, see [CDM05]). Let $\preceq_{O}^{\mathcal{D}}$ be the restriction of $\preceq_{O}$ to $\mathcal{D}$.

THEOREM 6. There is a continuous function $G \mapsto G^{*}$ assigning to each graph $G$ on $\mathbb{N}$ a dendrite $G^{*}$ in such a way that there is an embedding of $G$ into $H$ if and only if there is an open continuous surjection from $H^{*}$ onto $G^{*}$. Consequently, $\preceq_{O}^{\mathcal{D}}$ is a $\boldsymbol{\Sigma}_{1}^{1}$-universal preorder.

Proof. Let $\varrho_{n}$ be the prime number sequence. Let $X^{0}$ be a string; for $j \geq 1$ let $X^{j}$ be a $\varrho_{j}$-line, with all $X^{n} \subseteq[0,1]^{2}$, each $X^{n}$ having final point in $(1,1)$ and each $X^{j}$, with $j \geq 1$, having initial point $(0,0)$. Let also $X^{\infty}$ be a tame line with initial and final points $(0,0),(1,1)$, respectively. Now let $\left\{N_{t}\right\}_{t \in \mathbb{Z}<\omega}$ be a partition of $\mathbb{N}$ into two-element sets, with $N_{\emptyset}=\{0,1\}$. With the same conventions as in the proof of Theorem 3, if $N \subseteq N^{\prime} \subseteq \mathbb{N}$, the space $\mathbb{R}^{N}$ will be thought of as included in $\mathbb{R}^{N^{\prime}}$.

For any $j \in \mathbb{N} \cup\{\infty\}$ and $t \in \mathbb{Z}^{<\omega}$, let $X_{t}^{j} \subseteq[0,1]^{N_{t}}$ be the continuum obtained as a copy of $X^{j}$ by increasingly renaming the coordinates so that they belong to $N_{t}$. Let $q_{t}^{j}, p_{t}^{j}$ be the initial (if $j \neq 0$ ) and final point of $X_{t}^{j}$, respectively. These are the points with both coordinates 0 , respectively 1 , in the relevant square.

Each $t \in \mathbb{N}^{<\omega}$ will be called a good sequence; if some components of $t \in \mathbb{Z}^{<\omega}$ are negative, then $t$ will be called bad. Extend the definition of $\tau_{G}$ by letting $\tau_{G}(t)=\infty$ for $t$ a bad sequence.

To each graph $G$ on $\mathbb{N}$ we will associate in a continuous way a dendrite $G^{*}=\overline{\bigcup_{n \in \mathbb{N}} G_{n}}$, the closure of an increasing union of dendrites $G_{n}$, which, in turn, are defined inductively.

To begin with, define $G_{0}=X_{\emptyset}^{G}=X_{\emptyset}^{\tau_{G}(\emptyset)}=X_{\emptyset}^{0}$. Let $z_{\emptyset}^{G}$ be the final point of the string $X_{\emptyset}^{0}$ : its $N_{\emptyset}$ coordinates are $(1,1)$, all others are null. This is the point where the construction will grow at the next step. Call $z_{\emptyset}^{G}$ a good final point.

The next step is to define

$$
G_{1}=G_{0} \cup \bigcup_{i_{0} \in \mathbb{Z}} X_{i_{0}}^{G}, \quad \text { where } \quad X_{i_{0}}^{G}=\left\{z_{\emptyset}^{G}\right\} \times X_{i_{0}}^{\tau_{G}\left(i_{0}\right)} .
$$

For each $i_{0} \in \mathbb{Z}$, let $z_{i_{0}}^{G}$ be the final point of $X_{i_{0}}^{G}$ (these points have four coordinates 1 while all others are null). Call $z_{i_{0}}^{G} \operatorname{good}$ if $i_{0} \geq 0$, and bad otherwise. 
Next step:

$$
G_{2}=G_{1} \cup \bigcup_{\left(i_{0}, i_{1}\right) \in \mathbb{Z}^{2}} X_{\left(i_{0}, i_{1}\right)}^{G}, \quad \text { where } \quad X_{\left(i_{0}, i_{1}\right)}^{G}=\left\{z_{i_{0}}^{G}\right\} \times X_{\left(i_{0}, i_{1}\right)}^{\tau_{G}\left(i_{0}, i_{1}\right)} .
$$

Let $z_{\left(i_{0}, i_{1}\right)}^{G}$ be the final points of the lines $X_{\left(i_{0}, i_{1}\right)}^{G}$. Call a point $z_{\left(i_{0}, i_{1}\right)}^{G}$ good if $\left(i_{0}, i_{1}\right)$ is a good sequence, and bad otherwise.

In general, assuming $G_{n}$ built, let

$$
G_{n+1}=G_{n} \cup \bigcup_{t \in \mathbb{Z}^{n+1}} X_{t}^{G}, \quad \text { where } \quad X_{t}^{G}=\left\{z_{\left.t\right|_{n}}^{G}\right\} \times X_{t}^{\tau_{G}(t)} .
$$

If $z_{t}^{G}$ denotes the final point of the line $X_{t}^{G}$, call it good or bad according to the goodness of $t$.

Finally, let $z_{\xi}^{G}=\lim _{n \rightarrow \infty} z_{\left.\xi\right|_{n}}^{G}$ for $\xi \in \mathbb{Z}^{\mathbb{N}}$. So $G^{*} \backslash \bigcup_{n \in \mathbb{N}} G_{n}=\left\{z_{\xi}^{G}\right\}_{\xi \in \mathbb{Z}^{\mathbb{N}}}$.

The remark in the proof of Theorem 3 about the unnecessary use of the upper index $G$ in $z_{t}^{G}, z_{\xi}^{G}$ applies here as well.

By [N92, Theorem 10.36] the continuum $G^{*}$ is a dendrite, being homeomorphic to the inverse limit of the system $\left\{G_{n}, f_{n}\right\}_{n \in \mathbb{N}}$ where each bonding map $f_{n}: G_{n+1} \rightarrow G_{n}$ is the identity on $G_{n}$ and sends all points of each $X_{t}^{G}$ to $z_{\left.t\right|_{n}}^{G}$ for every $t \in \mathbb{Z}^{n+1}$, so it is monotone.

Note that each $X_{t}^{G} \backslash\left\{z_{t_{\text {length }(t)-1}^{G}}, z_{t}^{G}\right\}, t \neq \emptyset$, is open in $G^{*}$. Similarly, $X_{\emptyset}^{G} \backslash\left\{z_{\emptyset}^{G}\right\}$ is open. Also, each $z_{\xi}^{G}$, for $\xi$ an infinite sequence, has an open neighbourhood basis $\left\{U_{t}^{G}\right\}_{\emptyset \neq t \subset \xi}$, where

$$
U_{t}^{G}=\left(\bigcup_{t \subseteq s} X_{s}^{G} \cup\left\{z_{\zeta}^{G}\right\}_{t \subset \zeta}\right) \backslash\left\{z_{t_{\text {length }(t)-1}^{G}}\right\} .
$$

Each $U_{t}^{G}$ will be called the $t$-cone of $G^{*}$.

Let $G, H$ be graphs on $\mathbb{N}$.

Suppose $g: \mathbb{N} \rightarrow \mathbb{N}$ is an embedding of $G$ into $H$. We wish to define a continuous open surjection $h: H^{*} \rightarrow G^{*}$. Extend $g$ to a bijection $\gamma: \mathbb{Z} \rightarrow \mathbb{Z}$. If $g^{\prime}: \mathbb{N}^{<\omega} \cup \mathbb{N}^{\mathbb{N}} \rightarrow \mathbb{N}^{<\omega} \cup \mathbb{N}^{\mathbb{N}}$ is the injection induced by $g$ componentwise, then $\gamma$ induces componentwise a bijection $\gamma^{\prime}: \mathbb{Z}^{<\omega} \cup \mathbb{Z}^{\mathbb{N}} \rightarrow \mathbb{Z}^{<\omega} \cup \mathbb{Z}^{\mathbb{N}}$ which extends $g^{\prime}$. Let $s \in \mathbb{Z}^{<\omega}$ and let $t=\gamma^{\prime-1}(s)$. If $s$ is good and $s \in \operatorname{im} g^{\prime}$, then $t$ is good and $\tau_{G}(t)=\tau_{H}(s)$ : define $h$ on $X_{s}^{H}$ as a homeomorphism onto $X_{t}^{G}$ matching the final (and also initial, if $s \neq \emptyset$ ) point of $X_{s}^{H}$ with the final (respectively, the initial) point of $X_{t}^{G}$. If $s$ is bad or $s \notin \mathrm{im} g^{\prime}$, then $t$ is bad and so $X_{t}^{G}$ is a tame line. Define $h$ on $X_{s}^{H}$ as an open continuous function onto $X_{t}^{G}$ such that:

- the image of the initial (final, respectively) point of $X_{s}^{H}$ is the initial (final, respectively) point of $X_{t}^{G}$;

- the initial arc (final arc, $n$th bridge) of $X_{s}^{H}$ is mapped homeomorphically onto the initial arc (final arc, $n$th bridge) of $X_{t}^{G}$; 
- the $n$th bead of $X_{s}^{H}$ is mapped onto the $n$th bead of $X_{t}^{G}$ (note indeed that the $n$th bead in $X_{s}^{H}$ is homeomorphic to some $D_{i}$, while the $n$th bead in $X_{t}^{G}$ is homeomorphic to $D_{i^{\prime}}$ with $\left.i^{\prime} \leq i\right)$.

The definition of $h$ is then extended to $\left\{z_{\xi}^{H}\right\}_{\xi \in \mathbb{Z}^{\mathbb{N}}}$ by letting $h\left(z_{\xi}^{H}\right)=z_{\gamma^{\prime-1}(\xi)}^{G}$.

Claim. $h$ is continuous and onto.

Proof of claim. Since $h\left(H_{n}\right)=G_{n}$, let $h_{n}: H_{n} \rightarrow G_{n}$ be the restriction of $h$. If $\left\{G_{n}, f_{n}\right\}_{n \in \mathbb{N}},\left\{H_{n}, f_{n}^{\prime}\right\}_{n \in \mathbb{N}}$ are the inverse limits defining $G, H$ respectively, then $h_{n} f_{n}^{\prime}=f_{n} h_{n+1}$. Since each $h_{n}$ is continuous and onto, $h$ is continuous and onto as well by [N92, Exercise 2.22].

Claim. $h$ is open.

Proof of claim. It will be shown that $h$ is interior at each point $x \in H^{*}$ : for every open set $U$ in $H^{*}$ containing $x$, the point $h(x)$ is interior to $h(U)$. There are a few cases to be distinguished.

1) $x$ belongs to some string or line $X_{s}^{H}$ but is not an initial nor a final point of $X_{s}^{H}$. Then for each open neighbourhood $U$ of $x$ there is a smaller open neighbourhood $V$ of $x$ included in $X_{s}^{H}$. So $h(V)$ is an open subset of $G^{*}$ contained in some $X_{t}^{G}$.

2) $x=z_{s}^{H}$ for some $s \in \mathbb{Z}^{<\omega}$. Then for each open neighbourhood $U$ of $x$ there is a smaller open neighbourhood $V$ of $x$ which is the union of:

- $\left\{z_{s}^{H}\right\}$

- an open subarc of the final arc of $X_{s}^{H}$ having $z_{s}^{H}$ as one of its extrema;

- an open subarc of the initial arc of $X_{s^{\wedge} i}^{H}$, for $i$ ranging over a finite subset $A$ of $\mathbb{Z}$, having $z_{s}^{H}$ as one of its extrema;

- a union of cones $\bigcup_{i \in \mathbb{Z} \backslash A} U_{s^{\wedge} i}^{H}$.

Let $t=\gamma^{-1}(s)$. Then $h(V)$ is the union of:

- $\left\{z_{t}^{G}\right\}$

- an open subarc of the final arc of $X_{t}^{G}$ having $z_{t}^{G}$ as one of its extrema;

- an open subarc of the initial arc of $X_{t^{\wedge} i}^{G}$ for each $i \in \gamma^{-1}(A)$, having $z_{t}^{G}$ as one of its extrema;

- $\bigcup_{i \in \mathbb{Z} \backslash \gamma^{-1}(A)} U_{t^{\wedge} i}^{G}$.

So $h(V)$ is open in $G^{*}$.

3) $x=z_{\xi}^{H}$ for some $\xi \in \mathbb{Z}^{\mathbb{N}}$. Then each open neighbourhood $U$ of $x$ includes an $s$-cone containing $x$. The image under $h$ of an $s$-cone of $H^{*}$ is a $t$-cone of $G^{*}$ for some $t$.

Conversely, suppose $h: H^{*} \rightarrow G^{*}$ is an open continuous surjection. Recall the following properties of $h$ :

- $h$ maps end points of $H^{*}$ to end points of $G^{*}$; 
- the image under $h$ of a free arc in $H^{*}$ is a free arc in $G^{*}$ ([CCP94, Corollary 6.6]);

- $h$ is light (the preimage of each point is totally disconnected);

- $h$ preserves points of order $\aleph_{0}$;

- $h$ does not increase orders of points.

For each $s \in \mathbb{Z}^{<\omega}$, let

- $X_{s n}$ be the $n$th bead of $X_{s}^{H}$ and $Y_{s n}$ be the $n$th bead of $X_{s}^{G}$;

- $\alpha_{s n}$ be the $n$th bridge of $X_{s}^{H}$ and $\beta_{s n}$ be the $n$th bridge of $X_{s}^{G}$;

- $a_{s n}, b_{s n}$ be the first and second special points of $X_{s n}$ and $c_{s n}, d_{s n}$ be the first and second special points of $Y_{s n}$, so that $b_{s n}, a_{s, n+1}$ are the end points of $\alpha_{s n}$ and $d_{s n}, c_{s, n+1}$ are the end points of $\beta_{s n}$;

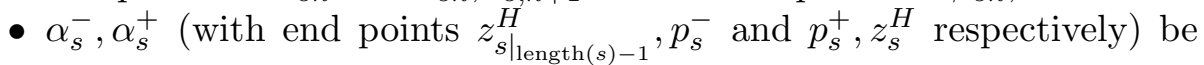
the initial and final arcs of $X_{s}^{H}$ and $\beta_{s}^{-}, \beta_{s}^{+}$(with respective end points

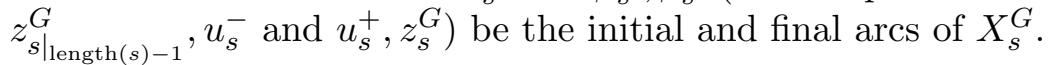

The above notations regarding initial points and arcs apply only for $s \neq \emptyset$. The following is proved by extending an idea from [CCP94].

Claim. $\forall s \in \mathbb{Z}^{<\omega} \exists t_{s} \in \mathbb{Z}^{<\omega}\left(h\left(X_{s}^{H}\right)=X_{t_{s}}^{G}\right)$, and the function $s \mapsto t_{s}$ preserves length and inclusion. Moreover, if $s$ is bad then so is $t_{s}$, while if $s, t_{s}$ are both good then $\tau_{G}\left(t_{s}\right)=\tau_{H}(s)$.

Proof of claim. Each bead of $H^{*}$ is a connected component of $\overline{E\left(H^{*}\right)}$ so it is mapped by $h$ onto a non-degenerate continuum with a dense set of end points. So $\forall(s, n) \exists(t, m) h\left(X_{s n}\right) \subseteq Y_{t m}$. This also implies that end points of bridges of $H^{*}$ are mapped to end points of bridges of $G^{*}$ and no non-special point of a bead in $H^{*}$ can be mapped to an end point of a bridge in $G^{*}$, since non-special points are interior to beads.

Let

$$
A=\left(X_{\emptyset 0} \cup \alpha_{\emptyset 0}\right) \backslash\left\{a_{\emptyset 1}\right\}=\left(X_{\emptyset 0} \backslash\left\{b_{\emptyset 0}\right\}\right) \cup\left\{b_{\emptyset 0}\right\} \cup\left(\alpha_{\emptyset 0} \backslash\left\{b_{\emptyset 0}, a_{\emptyset 1}\right\}\right) .
$$

So the open set $h(A)$ is the union of the open set $h\left(X_{\emptyset 0} \backslash\left\{b_{\emptyset 0}\right\}\right)$, the singleton $\left\{h\left(b_{\emptyset 0}\right)\right\}$ and the open free arc $h\left(\alpha_{\emptyset 0} \backslash\left\{b_{\emptyset 0}, a_{\emptyset 1}\right\}\right)$. It follows that $h\left(X_{\emptyset 0}\right)=$ $Y_{\emptyset 0}$ and $h\left(b_{\emptyset 0}\right)=d_{\emptyset 0}$ : if $h\left(X_{\emptyset 0}\right)$ were a proper subset of some $Y_{t m}$, take $y \neq h\left(b_{\emptyset 0}\right)$ on the boundary of $h\left(X_{\emptyset 0}\right)$ as a subset of $Y_{t m}$ and $x \in X_{\emptyset 0}$ such that $h(x)=y$. Then the image of an open subset $V$ of $H^{*}$ with $x \in V \subseteq X_{\emptyset 0}$ would lead to a contradiction. As $h\left(X_{\emptyset_{0}} \backslash\left\{b_{\emptyset 0}\right\}\right)=Y_{t m} \backslash\left\{h\left(b_{\emptyset 0}\right)\right\}$ is open in $G^{*}$, it follows that $(t, m)=(\emptyset, 0)$ and $h\left(b_{\emptyset 0}\right)=d_{\emptyset 0}$. Since $h\left(X_{\emptyset 1}\right)$ is a subset of some bead of $G^{*}$, it follows that $h\left(a_{\emptyset 1}\right) \in\left\{d_{\emptyset 0}, c_{\emptyset 1}\right\}$. However, if $h\left(a_{\emptyset 1}\right)=d_{\emptyset 0}$, then $h\left(\alpha_{\emptyset 0} \backslash\left\{b_{\emptyset 0}, a_{\emptyset 1}\right\}\right)$ would not be open. So $h\left(a_{\emptyset 1}\right)=c_{\emptyset 1}$ and $f\left(X_{\emptyset 1}\right) \subseteq Y_{\emptyset 1}$. 
Now suppose $(s, n) \neq(\emptyset, 0)$ and consider the open set

$$
\begin{aligned}
B= & \left(X_{s n} \cup \alpha_{s, n-1} \cup \alpha_{s n}\right) \backslash\left\{b_{s, n-1}, a_{s, n+1}\right\} \\
= & \left(\alpha_{s, n-1} \backslash\left\{b_{s, n-1}, a_{s n}\right\}\right) \cup\left\{a_{s n}\right\} \cup\left(X_{s n} \backslash\left\{a_{s n}, b_{s n}\right\}\right) \\
& \cup\left\{b_{s n}\right\} \cup\left(\alpha_{s n} \backslash\left\{b_{s n}, a_{s, n+1}\right\}\right) .
\end{aligned}
$$

Let $(t, m)$ be such that $h\left(X_{s n}\right) \subseteq Y_{t m}$. Note that $(t, m) \neq(\emptyset, 0)$, since all branching points of $Y_{\emptyset 0}$ have order 6 . Since $h\left(\alpha_{s, n-1}\right)$ and $h\left(\alpha_{s n}\right)$ are free arcs, it follows that $\left\{h\left(a_{s n}\right), h\left(b_{s n}\right)\right\} \subseteq\left\{c_{t m}, d_{t m}\right\}$. If $h\left(X_{s n}\right) \subset Y_{t m}$ let $y \notin\left\{c_{t m}, d_{t m}\right\}$ be a point of the boundary of $h\left(X_{s n}\right)$ as subset of $Y_{t m}$ and let $x \in X_{s n} \backslash\left\{a_{s n}, b_{s n}\right\}$ with $h(x)=y$. If $V$ is open in $H^{*}$ with $x \in V \subseteq X_{s n}$, then $y \in h(V) \subseteq h\left(X_{s n}\right)$, leading to a contradiction. So $h\left(X_{s n}\right)=Y_{t m}$ and $\left\{h\left(a_{s n}\right), h\left(b_{s n}\right)\right\}=\left\{c_{t m}, d_{t m}\right\}$. Since $h\left(X_{s, n-1}\right)$ and $h\left(X_{s, n+1}\right)$ are included in some beads, it follows that $h\left(b_{s, n-1}\right)$ and $h\left(a_{s, n+1}\right)$ are special points of some beads in $G^{*}$. However, $h\left(b_{s, n-1}\right) \neq h\left(a_{s n}\right)$, otherwise $h\left(\alpha_{s, n-1} \backslash\left\{b_{s, n-1}, a_{s n}\right\}\right)$ would not be open; similarly $h\left(a_{s, n+1}\right) \neq h\left(b_{s n}\right)$. So $h\left(X_{s, n-1}\right) \subseteq Y_{t, m \pm 1}$ and $h\left(X_{s, n+1}\right) \subseteq Y_{t, m \mp 1}$.

From this discussion it follows that:

(1) $\forall n \in \mathbb{N}\left(h\left(X_{\emptyset n}\right)=Y_{\emptyset n}\right)$;

(2) $h\left(p_{\emptyset}^{+}\right)=u_{\emptyset}^{+}$;

(3) $h\left(z_{\emptyset}^{H}\right)=z_{\emptyset}^{G}\left(\right.$ since ord $\left.\left(z_{\emptyset}^{H}, H^{*}\right)=\aleph_{0}\right)$;

(4) $\forall s \in \mathbb{Z}^{<\omega} \backslash\{\emptyset\} \exists t_{s} \in \mathbb{Z}^{<\omega} \backslash\{\emptyset\} \exists k \in \mathbb{Z} \forall n \in \mathbb{Z}\left(h\left(X_{s n}\right)=Y_{t_{s}, k \pm n}\right)$.

Actually in (4) the only consistent possibility is

$\left(4^{\prime}\right) \exists k \in \mathbb{Z} \forall n \in \mathbb{Z}\left(h\left(X_{s n}\right)=Y_{t_{s}, k+n}\right)$,

since otherwise beads homeomorphic to $D_{3}$ would be mapped eventually onto beads $D_{i}$ with $i>3$. This argument also shows that if $s$ is bad then so is $t_{s}$, and that if $s$ is good then either $t_{s}$ is bad or $\tau_{G}\left(t_{s}\right)=\tau_{H}(s)$. Now it will be argued inductively that $s \mapsto t_{s}$ preserves length and inclusion. Indeed, note that from $h\left(z_{\emptyset}^{H}\right)=z_{\emptyset}^{G}$ this follows for $s$ of length 1, implying also $h\left(z_{s}^{H}\right)=z_{t_{s}}^{G}$. Given $h\left(z_{s}^{H}\right)=z_{t_{s}}^{G}$ the assertion follows for all extensions $s^{\frown} i$ of $s$ by applying $\left(4^{\prime}\right)$ to $s^{\frown} i$.

By continuity and surjectivity of $h$, it follows that $h\left(\left\{z_{\xi}^{H}\right\}_{\xi \in \mathbb{Z}^{\mathbb{N}}}\right)=$

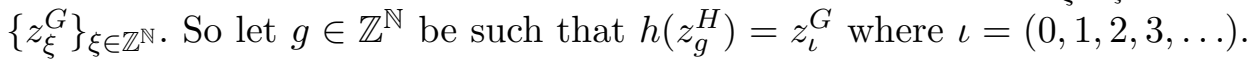
Then $h\left(X_{\left.g\right|_{n}}^{H}\right)=X_{(0,1, \ldots, n-1)}^{G}$ for all $n \in \mathbb{N}$. Thus each $\left.g\right|_{n}$ is a good sequence and $\tau_{H}\left(\left.g\right|_{n}\right)=\tau_{G}(0,1, \ldots, n-1)$; so $g$ is an embedding from $G$ into $H$.

REMARK. With an argument similar to the one used in Remark 2 of Section 2, the above proof can be modified to produce planar dendrites. 


\section{References}

[Ca] R. Camerlo, Universality of embeddability relations for coloured total orders, Order, to appear.

[CDM05] R. Camerlo, U. B. Darji and A. Marcone, Classification problems in continuum theory, Trans. Amer. Math. Soc. 357 (2005), 4301-4328.

[Ch80] J. J. Charatonik, Open mappings of universal dendrites, Bull. Acad. Polon. Sci. Sér. Sci. Math. 28 (1980), 489-494.

[CCP94] J. J. Charatonik, W. J. Charatonik and J. R. Prajs, Mapping hierarchy for dendrites, Dissertationes Math. 333 (1994).

[E35] S. Eilenberg, Sur les transformations d'espaces métriques en circonférence, Fund. Math. 24 (1935), 160-176.

[FS89] H. Friedman and L. Stanley, A Borel reducibility theory for classes of countable structures, J. Symbolic Logic 54 (1989), 894-914.

[K95] A. S. Kechris, Classical Descriptive Set Theory, Springer, 1995.

[LR05] A. Louveau and C. Rosendal, Complete analytic equivalence relations, Trans. Amer. Math. Soc. 357 (2005), 4839-4866.

[Mać86] T. Maćkowiak, Singular arc-like continua, Dissertationes Math. 257 (1986).

[Mar] A. Marcone, Complexity of sets and binary relations in continuum theory: a survey, preprint.

[MR04] A. Marcone and C. Rosendal, The complexity of continuous embeddability between dendrites, J. Symbolic Logic 69 (2004), 663-673.

[Me67] K. Menger, Kurventheorie, Chelsea, 1967.

[N92] S. B. Nadler, Jr., Continuum Theory, Dekker, 1992.

Dipartimento di Matematica

Politecnico di Torino

Corso Duca degli Abruzzi 24

10129 Torino, Italy

E-mail: camerlo@calvino.polito.it

Received 30 December 2004;

in revised form 18 August 2005 